\title{
Hot biopsy forceps in the diagnosis of endobronchial lesions
}

\author{
A. Tremblay*,\#, G. Michaud* and S.J. Urbanski ${ }^{\mp}$
}

\author{
ABSTRACT: Electrocoagulation bronchoscopy biopsy forceps may prevent bleeding, but could \\ also impair the quality of the specimens obtained.
}

Patients with endobronchial lesions during bronchoscopy underwent six endobronchial biopsies each with a hot biopsy forceps, alternating between with electrocoagulation ("hot") and without ("cold"). Bleeding was quantified on a scale of 1-4, with 1 being no bleeding. The generator was set on "soft coagulation" mode, with power settings of 40, 60, 80 and $100 \mathrm{~W}$ for each group of 10 patients in a sequential fashion. Clinical pathology results were recorded before samples were reviewed by a second, blinded, pulmonary pathologist.

A total of $\mathbf{3 9}$ patients with $\mathbf{4 0}$ endobronchial lesions had six biopsies performed (one patient had only four samples taken), giving a total of 238 biopsy samples. Concordance between hot and cold samples was $\mathbf{9 2 . 5 \%}$ for the clinical pathologist and $\mathbf{8 7 \%}$ for the blinded pathologist. Paired analysis suggested lower average bleeding score with the use of hot forceps. Overall bleeding rates for cold and hot biopsies, respectively, were as follows: grade 1: 30.3 and 41.2\%; grade 2: 62.2 and 49.6\%; grade 3: 7.6 and $9.2 \%$; and grade $4: 0$ and $0 \%$.

In conclusion, the use of hot biopsy forceps for endobronchial biopsy does not appear to have a negative impact on the pathological samples. Hot biopsy forceps showed a statistically significant reduction in bleeding score, which is unlikely to be of clinical significance.

\section{KEYWORDS: Biopsy, bronchoscopy, lung cancer}

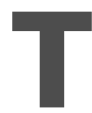
he traditional approach to diagnosis of endobronchial lesions relies on forceps biopsy during flexible bronchoscopic examination. A new electrocautery "hot" bronchoscopy biopsy forceps is now commercially available and may prevent bleeding following biopsy. Unfortunately, no published data exist regarding the use of this device.

The primary objective of the present study was to determine the impact of electrocautery forceps on pathological diagnosis following biopsy of endobronchial lesions. The secondary objective was to comparebleeding rates between the two techniques.

\section{METHODS}

The present study was conducted as a prospective controlled study, with each patient serving as his or her own control. The study protocol was approved by the Conjoint Health Research Ethics Board of the University of Calgary (Calgary, AB, Canada). Written informed consent was obtained for all patients included in the study.

\section{Inclusion/exclusion criteria}

Patients aged $>18$ yrs with a visible endobronchial lesion at flexible bronchoscopy performed by one of the investigators, and in whom biopsy was indicated for diagnosis of the lesion, were eligible for the study. Patients were excluded if they had a known or suspected bleeding disorder, or a cardiac pacemaker or implantable defibrillator in place.

\section{Procedure}

Patients underwent flexible bronchoscopy on an outpatient basis under conscious sedation with an opiate (morphine or fentanyl) and/or benzodiazepine (midazolam) administered intravenously, as well as topical anaesthesia. All patients had i.v. lines in place, as well as supplemental oxygen by mask or nasal canullae. All patients were monitored with ECG, automated blood pressure cuff and pulse oximetry. Patients were monitored post-procedure for a minimum period of $2 \mathrm{~h}$.

Once a suitable lesion was identified, six biopsies were taken, alternating between "hot" and "cold" without specifying the method for the initial biopsy. The samples were placed in two separate formalin containers. Quantification of bleeding was carried out and recorded by the bronchoscopist (unblinded to biopsy method)
AFFILIATIONS

*Divisions of Respiratory Medicine,

Thoracic Surgery and Medical

Oncology, and

-Dept of Pathology and Laboratory

Medicine, University of Calgary, and

\# Southern Alberta Cancer Research

Institute, Calgary, AB, Canada.

\section{CORRESPONDENCE}

A. Tremblay

Division of Respiratory Medicine

Health Sciences Center

3330 Hospital Drive NW

Calgary

$A B, T 2 N 4 N 1$

Canada

Fax: 14039441577

E-mail: alain.tremblay@ucalgary.ca

Received:

May 302006

Accepted after revision:

September 142006

SUPPORT STATEMENT

No conflict of interest exists for any of the authors with regard to the subject matter of this manuscript. 
after each biopsy according to a four-point scale (1: no bleeding; 2: minimal bleeding not requiring intervention; 3: mild-to-moderate bleeding requiring intervention, such as icecold saline, topical adrenaline or electrocoagulation; 4: moderate-to-severe bleeding requiring termination of procedure, endotracheal intubation or other invasive treatment). Additional biopsies or other sampling were performed at the discretion of the bronchoscopist, but were placed in separate containers.

All biopsies were taken with an electrocoagulation-capable biopsy forceps (FD-6C-1A; Olympus America, Melville, NY, USA) with ("hot") or without ("cold") the application of an electrocoagulation current (ICC 350 electrosurgical generator; ERBE, Tübingen, Germany) set on "soft coagulation" mode. The power setting on the generator was set at 40,60, 80 and $100 \mathrm{~W}$ for each group of 10 patients, in a sequential fashion.

\section{Pathology}

All biopsy samples were sent to the pathology department, without any identification of treatment group, for interpretation by the clinical pathologist assigned to the case. The diagnosis reported by the clinical pathologist was recorded for each sample. Once patient enrolment was completed, a research pathologist blinded to treatment group reviewed all samples without knowledge of the clinical pathology report. Only one set of the biopsy pair from a given patient was reviewed at a given time. The research pathologist recorded a pathological diagnosis for each set of samples and was asked to grade the quality of the sample with regards to electrocoagulation damage according to a three-point scale (1: no or mild damage; 2 : moderate damage; 3 : severe damage).

If the endobronchial biopsies were nondiagnostic, other samples taken at the time of bronchoscopy or additional samples taken at a later date were reviewed and recorded for study purposes.

\section{Analysis}

All patients with documented endobronchial lesions who underwent at least two biopsies (one with and one without electrocoagulation) were included in the analysis.

The primary outcome measure for the study was the discordance of pathological results obtained with the hot and cold methods. A McNemar's test for changes to a $4 \times 4$ table was applied comparing results from both diagnostic techniques. Fisher's exact test was also used to compare the diagnostic yield between the two approaches and a $95 \%$ confidence interval of the frequency of positive concordance between the two approaches was calculated.

The study sample size of 40 patients was calculated using Fisher's exact test (two-sided) as if the samples were unmatched, with a power of 0.80 to detect a decrease in diagnostic yield from $95 \%$ to $70 \%$, with an alpha of 0.05 . This approach was used as there was no way to determine the expected frequency of discordant results between each diagnostic test required for the McNemar's test to be used in sample size calculations. Ten discordant results would be required to show the above difference with the McNemar's test.
Comparison of bleeding rates was performed by comparing the average bleeding score for hot versus cold biopsies in a paired nonparametric analysis (Wilcoxon signed rank tests).

\section{RESULTS}

A total of 39 patients with 40 endobronchial lesions were entered into the study and had the six biopsies performed as per protocol (one patient had only four samples taken), giving a total of 238 biopsy samples. Of the subjects, $56 \%$ were male (22 out of 39). The mean (range) age was 60.8 yrs (20-85).

Locations of biopsies and final diagnoses are listed in table 1. A diagnosis was made with endobronchial biopsy in 35 out of 40 cases $(87.5 \%)$.

Concordance between hot and cold samples was $92.5 \%$ for clinical pathologist and $87 \%$ for research pathologist $(\mathrm{p}>0.05$, McNemar's test), suggesting lack of significant impact of hot biopsy on diagnostic yield (table 2). The degree of electrocoagulation damage was graded as 1 (minimal) for all samples.

Paired analysis of the average bleeding score between hot and cold biopsies showed a statistically significant lower bleeding score for hot samples $(p=0.03)$. Overall bleeding rates for cold and hot biopsies were, respectively, as follows: grade 1: 30.3 and $41.2 \%$; grade 2: 62.2 and $49.6 \%$; grade $3: 7.6$ and $9.2 \%$; and grade $4: 0$ and $0 \%$ ( $p>0.05$; Kruskal-Wallis test; fig. 1a). No significant difference in bleeding was seen when data were analysed according to power level (fig. 1b). This suggests that the main effect of the hot biopsy on bleeding is a reduction in the number of mild bleeding episodes, but that a reduction in moderate bleeding is not seen. No cases of severe bleeding were seen during the study period.

\section{DISCUSSION}

The diagnostic yield following endobronchial biopsy is excellent, with the largest case series demonstrating sensitivities ranging $76-97 \%[1,2]$. It has also been demonstrated that three samples are sufficient to maximise diagnostic yield [3],

$\begin{array}{lr}\text { TABLE } 1 \quad \begin{array}{l}\text { Locations of lesions and final diagnoses in } \\ \text { patients }\end{array} \\ \begin{array}{l}\text { Location of lesion \% } \\ \text { Right upper lobe }\end{array} \\ \text { Right mainstem } & 24.4 \\ \text { Left mainstem } & 15.1 \\ \text { Left upper lobe } & 12.6 \\ \text { Right lower lobe } & 12.6 \\ \text { Left lower lobe } & 12.6 \\ \text { Right middle lobe } & 7.6 \\ \text { Trachea } & 5.0 \\ \text { Bronchus intermedius } & 5.0 \\ \text { Final diagnosis \% } & 5.0 \\ \text { Nonsmall cell carcinoma } & \\ \text { Lymphoma } & 65.0 \\ \text { Small cell carcinoma } & 12.5 \\ \text { Carcinoid tumour } & 7.5 \\ \text { Renal cell carcinoma } & 7.5 \\ \text { Granular cell tumour } & 2.5 \\ \text { Hamartoma } & 2.5 \\ \end{array}$




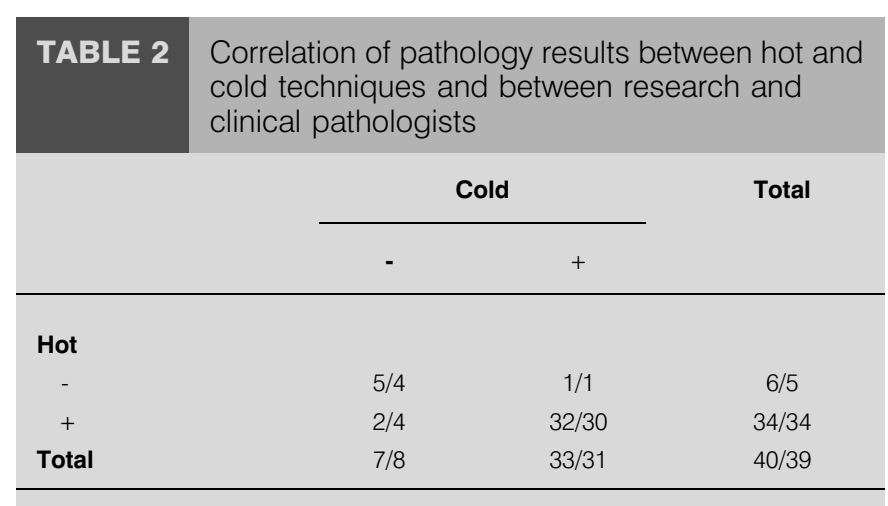

Data are presented as research/clinical. One sample was unavailable for review by the research pathologist. -: no diagnosis; +: diagnosis.
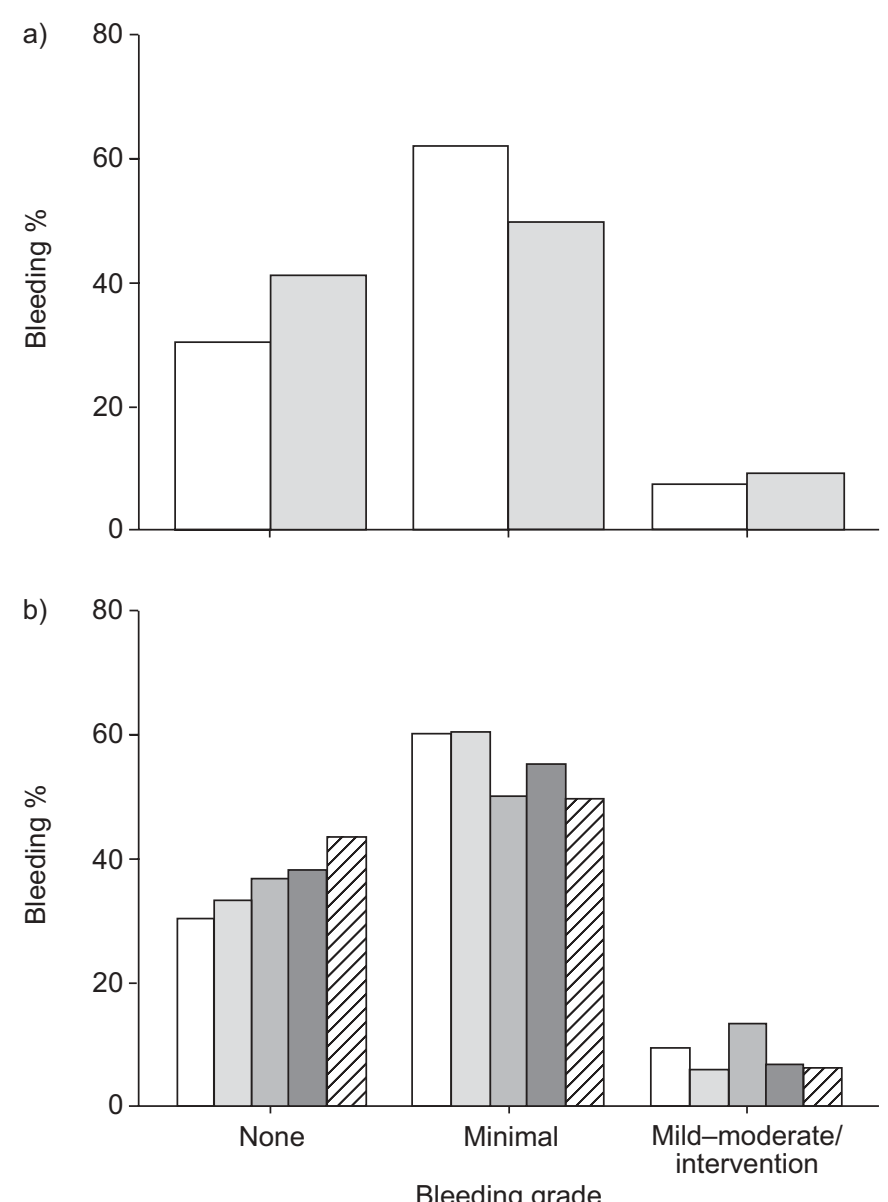

FIGURE 1. Bleeding rates according to a) use of electrocoagulation ( $\square$ : electrocoagulation used; $\square$ : electrocoagulation not used; and b) power setting ( $\square$ : o W; $: 40 \mathrm{~W} ; \quad: 60 \mathrm{~W} ; \mathbf{\square}: 80 \mathrm{~W} ; \mathbb{Z}: 100 \mathrm{~W}$ ).

although the collection of additional samples has been justified by others $[4,5]$.

The only significant complication following endobronchial biopsy is bleeding. Mild, self-limited bleeding is common [6] and may lead to difficult visualisation, potentially impeding the remainder of the examination. Severe life-threatening bleeding is fortunately a rare event. One report described two deaths attributed to bleeding in a questionnaire study of $\sim 48,000$ bronchoscopy procedures [7]. Brisk bleeding was seen in six out of 330 patients reported in a second series but no fatalities occurred [1].

Endobronchial electrocoagulation is an effective tool in the management of endobronchial lesions and can be very effective at controlling bleeding from an identified bleeding site [8-10]. This makes new biopsy forceps capable of applying an electrocoagulation current at the time of biopsy promising in terms of reducing bleeding risk following this common procedure. Despite the commercial availability of such a forceps, no published investigations address the potential impact of this approach on the quality of the biopsy specimen obtained, despite the fact that electrocautery applied to the airway can lead to significant pathological changes in the tissues $[11,12]$. Nor has the impact of hot forceps on bleeding rate been reported.

The present study was performed to better understand the performance characteristics of a new commercially available device designed to obtain endobronchial biopsies with simultaneous application of an electrocautery current aimed at preventing or reducing bleeding. The study was powered to detect a significant impact on the diagnostic yield of the samples, as it was felt that a greater harm could come to patients if this device lowered the diagnostic yield from bronchoscopy biopsy than if the device simply failed to prevent bleeding.

Fortunately, the hot biopsy forceps did not appear to impair the quality of the specimens obtained or hinder the pathologist's ability to make a diagnosis.

The next issue was to determine whether the device actually does what it is designed to do: reduce bleeding rates. Although a statistically significant decrease in mean bleeding score was noted with the use of the electrocoagulation current, this seemed to be limited to a reduction in the number of minor bleeding episodes (grade 2), which are unlikely to be of clinical significance. No decrease in moderate bleeding was noted, although the number of these events was low and a larger study would be needed to have sufficient power to confirm this finding. Similarly, severe bleeding episodes were rare enough that it would be difficult to show a significant difference in this outcome. Given that the population studied did not have any particular increased risk for bleeding, the present authors cannot exclude the possibility that this technique may reduce bleeding in high-risk patients or lesions. During the study period, only one patient had severe bleeding resulting in termination of the procedure. Interestingly, this occurred during a hot biopsy, but the patient was not included in the study as per protocol, given that no matching cold biopsy was obtained.

The lack of blinding at the time of quantification of bleeding could potentially bias the results of the study, but this is difficult to remedy. The use of a simple scoring system and the initial equipoise of the current investigators regarding the effect of this device should have minimised any bias. Another potential bias relates to the lack of randomisation of the sequence of the biopsies. Given an alternating biopsy protocol 
(i.e. cold-hot-cold-hot-cold-hot or vice versa), it seems unlikely that starting with one approach rather than the other would impact bleeding rates or the quality of the samples.

The cost of the biopsy forceps is relatively minor, as it is a reusable device; however, the infection-control implications of reusable biopsy forceps need to be considered. The cost of the electrosurgical generator is much more important, but such devices may be easily accessible in most hospitals.

Given these findings, it would not seem that routine use of the electrocoagulation bronchoscopy forceps is warranted. The present authors have occasionally found it useful to apply electrocautery when moderate bleeding occurs following endobronchial biopsy. This can be done using the biopsy forceps as the electrocautery probe and is of course facilitated if all the equipment is already set up. Familiarity with this equipment is also important and may lead to improved patient and bronchoscope safety. Other simpler methods, such as topical application of ice-cold saline or adrenaline, may be easier to apply and more easily accessible to bronchoscopists.

\section{ACKNOWLEDGEMENTS}

The authors wish to thank G. Fick, the Respiratory Therapy Dept at Foothills Medical Center (Calgary, AB, Canada) and D. Moise for their assistance with this study.

\section{REFERENCES}

1 Zavala DC. Diagnostic fiberoptic bronchoscopy: techniques and results of biopsy in 600 patients. Chest 1975; 68: 12-19.

2 Mak VH, Johnston ID, Hetzel MR, Grubb C. Value of washings and brushings at fibreoptic bronchoscopy in the diagnosis of lung cancer. Thorax 1990; 45: 373-376.

3 Shure D, Astarita RW. Bronchogenic carcinoma presenting as an endobronchial mass. Chest 1983; 83: 865-867.
4 Gellert AR, Rudd RM, Sinha G, Geddes DM. Fibreoptic bronchoscopy: effect of experience of operator on diagnostic yield of bronchial biopsy in bronchial carcinoma. $\mathrm{Br}$ J Dis Chest 1982; 76: 397-399.

5 Popovich J Jr, Kvale PA, Eichenhorn MS, Radke JR, Ohorodnik JM, Fine G. Diagnostic accuracy of multiple biopsies from flexible fiberoptic bronchoscopy. A comparison of central versus peripheral carcinoma. Am Rev Respir Dis 1982; 125: 521-523.

6 Bilaceroglu S, Gunel O, Cagirici U, Perim K. Comparison of endobronchial needle aspiration with forceps and brush biopsies in the diagnosis of endobronchial lung cancer. Monaldi Arch Chest Dis 1997; 52: 13-17.

7 Suratt PM, Smiddy JF, Gruber B. Deaths and complications associated with fiberoptic bronchoscopy. Chest 1976; 69: 747-751.

8 Tremblay A, Marquette CH. Endobronchial electrocautery and argon plasma coagulation: a practical approach. Can Respir J 2004; 11: 305-310.

9 Reichle G, Freitag L, Kullmann HJ, Prenzel R, Macha HM, Farin G. Argon plasma coagulation in bronchology: a new method - alternative or complementary? Pneumologie 2000; 54: 508-516.

10 Morice RC, Ece T, Ece F, Keus L. Endobronchial argon plasma coagulation for treatment of hemoptysis and neoplastic airway obstruction. Chest 2001; 119: 781-787.

11 Verkindre C, Brichet A, Maurage CA, Ramon P, Homasson $\mathrm{JP}$, Marquette $\mathrm{CH}$. Morphological changes induced by extensive endobronchial electrocautery. Eur Respir J 1999; 14: 796-799.

12 van Boxem TJ, Westerga J, Venmans BJ, Postmus PE, Sudetja TG. Tissue effects of bronchoscopic electrocautery: bronchoscopic appearance and histologic changes of bronchial wall after electrocautery. Chest 2000; 117: 887-891. 